\title{
Recovery from first-episode psychosis: early identification and prompt initiation of treatment improve long-term outcomes
}

\author{
Angus MacBeth \\ Psychiatry Research Group, Clinical Research Centre, University of Aberdeen \& NHS Grampian, Aberdeen, UK
}

\section{WHAT IS ALREADY KNOWN ON THIS TOPIC?}

Improving recovery (ie, sustained symptomatic and functional remission) remains a challenge in the treatment of schizophrenia. Evidence suggests that the median recovery rate for schizophrenia is $13.5 \%{ }^{1}$ Improving our understanding of predictors of recovery, particularly from first-episode psychosis (FEP), could enable better specification of treatment approaches relevant to individual presentation and illness stage. The OPUS trial is one of the largest prospective cohort studies of individuals receiving treatment for FEP to date. Participants were recruited from the community and received either a specialised assertive outreach approach for FEP or treatment as usual.

\section{WHAT THIS PAPER ADDS?}

- At 10 -year follow-up, $25 \%$ of individuals displayed sustained positive and negative symptomatic remission, with $14 \%$ of the sample achieving full functional and symptomatic recovery. These data are broadly consistent with existing recovery rates. ${ }^{2}$

- After controlling for associations between predictors, lower levels of negative symptoms and younger age predicted recovery at 10-year follow-up. This suggests that accurate early identification of psychosis in young adults and prompt initiation of treatment, particularly approaches targeting negative symptoms, can confer long-term benefits for outcome.

\section{LIMITATIONS}

- Findings are reported for the whole study sample, thus results for individuals receiving specialised FEP treatment are combined with those receiving treatment as usual. Therefore, it is not possible to evaluate whether the type of treatment received affected recovery rates.

- About $40 \%$ of the original study sample was lost to follow-up. This group may represent hard-to-engage patients. Effective treatment planning, when patients are stable and in crisis, requires collaboration between patient and clinical team; therefore, hard-to-engage patients are frequently of concern to teams. Our knowledge of this group's treatment needs remains sparse.

- The study was conducted in a public healthcare setting; consequently, results may not generalise to other models of healthcare provision.

\section{WHAT NEXT IN RESEARCH?}

Further reports of long-term outcomes for prospective FEP cohorts are needed. In particular, findings for the contribution of evidence-based interventions - such as cognitive behavioural therapy or supported employment programmes - to recovery rates would be welcomed.

\section{DO THESE RESULTS CHANGE YOUR PRACTICES AND WHY?}

The study supports an increasing body of evidence highlighting the continued need for early, accurate identification of $\mathrm{FEP}^{3}$ The trial also emphasises that integrative treatment models are needed to improve outcomes for the functional and symptomatic aspects of recovery, incorporating pharmacological and psychological interventions alongside interventions that support individuals back into education, training or employment. For practitioners working as part of specialised multiprofessional early intervention teams, the observation that these factors may be implicated in recovery will not come as a surprise. However, for those working in generic mental health teams, the findings highlight the continued need to look beyond 'one size fits all' routine care and crisis management in treating psychosis.

Competing interests None.

doi:10.1136/eb-2014-101766

\section{REFERENCES}

1. Jääskeläinen $\mathbf{E}$, Juola $\mathrm{P}$, Hirvonen $\mathrm{N}$, et al. A systematic review and meta-analysis of recovery in schizophrenia. Schizophr Bull 2013;39:1296-306.

2. Harrison G, Hopper K, Craig T, et al. Recovery from psychotic illness: a 15- and 25-year international follow-up study. Br J Psychiatry 2001;178:506-17.

3. Birchwood M, Connor $\mathrm{C}$, Lester $\mathrm{H}$, et al. Reducing duration of untreated psychosis: care pathways to early intervention in psychosis services. Br J Psychiatry 2013;203:58-64. 
ABSTRACT FROM: Austin SF, Mors O, Secher RG, et al. Predictors of recovery in first episode psychosis: the OPUS cohort at 10 year follow-up. Schizophr Res 2013;150:163-8.

Patients/participants Participants with first-episode psychosis who had a diagnosis of schizophrenia spectrum disorder (International Classification of Diseases (ICD) 10: block F20-29), had not received more than 12 weeks of antipsychotic medication, and were aged between 18 and 45 years. People with schizotypal disorder (F21) were excluded because they do not experience psychotic symptoms. All participants were recruited from the outcomes following vaginal prolapse repair and mid-urethral sling (OPUS) trial.

Setting Centres in Denmark (Copenhagen and Aarhus); 1998-2000 (baseline).

Prognostic factors Severity of positive and negative symptoms (schedules for positive and negative symptoms); course of illness over the past 2 years; level of functioning (Global Assessment of Functioning scale (GAF)); premorbid functioning (Premorbid Adjustment scale); duration of untreated psychosis (interview for retrospective assessment of onset of schizophrenia); days of hospitalisation; number of contacts with psychiatric services; number of days in supported housing.

Control None.

Follow-up period 10 years. Follow-up rate at 10 years was $61 \%$ (304 of 496).

\section{OUTCOMES}

\section{Recovery after 10 years}

42 of 304 (14\%) participants who completed follow-up met the criteria for full recovery at 10 years (defined as stable remission of negative and positive symptoms, no psychiatric admissions to hospital or living in supported accommodation for the past 2 years, currently engaged in work or study and a GAF score of over 60).

\section{Rates of symptom remission and full recovery}

Rates of full recovery appeared stable at 2, 5 and 10 years; however, not the same people achieved remission or recovery at each follow-up. Overall, 64\% participants achieved symptom remission at least once, and nearly $30 \%$ achieved full recovery at some point during the 10 years.

\section{Predictors of recovery}

Baseline predictors accounted for $22 \%$ of the variance in rates of full recovery. Lower severity of negative symptoms at baseline (OR $0.53,95 \%$ CI 0.36 to 0.78 ) and earlier age at diagnosis (OR 0.92 , $95 \%$ CI 0.86 to 0.99 ) were predictive of recovery at 10 years. At 1-year follow-up, negative symptoms were found to independently predict recovery at 10 years (OR $0.49,95 \%$ CI 0.25 to 0.98 ). 